\title{
Estudo comparativo entre diagnósticos radiográficos obtidos pelas técnicas interproximal e panorâmica*
}

\author{
Comparative study of radiographic diagnostics observed in bitewing \\ and panoramic techniques.
}

Reni Raymundo Dalla-Bona** Vania Fontanella*** Aderson Gegler****

\begin{abstract}
RESUMO
Foram comparados os diagnósticos radiográficos obtidos pelas técnicas interproximal e panorâmica na área de abrangência da primeira, bem como avaliados os dignósticos adicionais fornecidos pela última, em uma amostra constituída por radiografias de 50 pacientes adultos, examinadas pelo mesmo observador. A proporção de diagnósticos coincidentes foi significativamente maior que a de diagnósticos divergentes, sugerindo não existir diferença entre o rendimento diagnóstico de radiografias panorâmica e interproximais, na área de abrangência da última. Além da área de abrangência das radiografias interproximais, a panorâmica mostrou, para a média dos pacientes, 3,72 alterações que requereriam tratamento ou controle.
\end{abstract}

\section{Unitermos \\ Radiologia odontológica - Radiografia interproximal - Radiografia panorâmica}

\section{INTRODUÇÃO}

O uso dos $\mathrm{Rx}$ com propósitos diagnósticos tem sido bem estabelecido desde sua descoberta. $O$ alerta sobre o perigo da radiação $X$, seus efeitos e sugestões de proteção, aparecem na literatura odontológica desde 1914. No entanto, o reconhecimento coletivo pelos profissionais da real necessidade de proteção contra a radiação veio bem mais tarde. (38)

Os pacientes odontológicos têm sido examinados radiograficamente através de incidências panorâmica, periapicais e interproximais ou uma combinação destes três métodos radiográficos. A radiografia panorâmica utiliza écrans intensificadores e requer menos radiação que algumas poucas radiografias intrabucais. Entretanto, apresenta a desvantagem de mostrar menor definição e detalhe, se comparada a radiografias convencionais intrabucais. (24) Dependendo do tipo de informação desejada, um método radiográfico pode ser o escolhido, ou vários exames devem ser empregados. (35)

Na prática da radiologia odontológica se observa que os cirurgiões-dentistas de nosso meio se valem de todas as combinações possíveis de radiografias para a complementação do exame clínico de um novo paciente. Vale salientar que, enquanto alguns solicitam inicialmente somente uma radiografia panorâmica ou somente as interproximais, outros solicitam ambas as técnicas e, por vezes, a solicitação é de panorâmica, exame periapical completo e interproximais.

É uma preocupação mundial encontrar um protocolo de exames radiográficos que complemente o exame clínico e que revele o maior número de dados possíveis para um completo diagnóstico e ainda resulte em uma menor dose de exposição à radiação e em menor custo financeiro. Muitos trabalhos existem nesta área, no entanto, vários se complementam, alguns se equivalem e outros se contrapõem. (53)

\section{REVISÃO DA LITERATURA}

A dose-equivalente de uma radiografia panorâmica estimada em $80 \mathrm{mmSv}$ no início dos anos 80 (64) diminuiu para $6,7 \mathrm{mmSv}$, devido às modificações nos equipamento e à utilização de novos écrans e filmes mais sensiveis, diminuindo o risco estimado de câncer de $1,3 \times 10^{-6}$ para $0,21 \times 10^{-16}$.(66)

$\mathrm{Um}$ exame radiográfico intrabucal completo é demorado, expõe o paciente a uma dose relativamente alta de radiação e é ocasionalmente associado com desconforto ao paciente. A radiação recebida em uma radiografia panorâmica é relativamente baixa quando comparada ao exame radiográfico intrabucal completo. (37)

Vários autores concordam que a qualidade da imagem está relacionada intimamente aos cuidados técnicos na exposição e processamento radiográficos, salientando que quando o exame é realizado por técnico treinado os erros diminuem drasticamente. $(4,11,55,56)$

Devido à ampla área abrangida pela panorâmica, particularmente os arcos zigomáticos, maxila, mandíbula e estruturas periféricas, essa técnica tornou-se popular e valiosa, entretanto apresenta magnificação e retrata distorção. Ela tem sido utilizada como rotina em diversas instituições e serviços privados. (5)

Vários trabalhos concluem que a radiografia panorâmica apresenta desempenho particularmente insatisfatório na evidenciação de cáries restritas ao esmalte, sendo mais efetiva para o diagnóstico de lesões extensas. $(1,7,24,37)$

Lesões de cárie nos dentes anteriores também não são bem demonstradas pela radiografia panorâmica $(24,43,59)$ técnica que apresenta sensibilidade variável para cárie de acordo com a região: $30 \%$ para molares, $19 \%$ para pré-molares e $8 \%$ para dentes anteriores. $(17,37)$

A panorâmica tem importante papel

\footnotetext{
* Resumo da dissertação apresentada como parte dos requisitos obrigatórios para obtenção do título de mestre em odontologia, na área de concentração clínica odontológica.

** Mestre em Odontologia pela FO-UFRGS; Prof. do Departamento de Cirurgia e Ortopedia da FO-UFRGS, Disciplinas de Radiologia. *** Doutora em Odontologia pela FO-PUCRS; Profa. do Departamento de Cirurgia e Ortopedia da FO-UFRGS, Disciplinas de Radiologia. **** Cirurgião-dentista; ex-bolsita do Programa de Iniciação Científica PIBIC-CNPq.
} 
no diagnóstico e manejo da doença periodontal pois evidencia perda óssea, aumento do espaço periodontal, identifica sinais de trauma oclusal, presença de tártaro, excessos de restaurações, má-adaptação de coroas protéticas e a proporção coroa/raiz. (22)

Radiografias interproximais são comumente utilizadas para mostrar perdas ósseas alveolares (44), contudo, ainda se encontram controvérsias na literatura em relação à eficácia da panorâmica no exame radiográfico da crista alveolar.

A radiografia panorâmica é mais fiel que as periapicais na determinação da altura da crista alveolar em crânios secos (2) e , por ser reprodutível, é válida na investigação do padrão de perda óssea alveolar na doença periodontal. (27) Contudo, DOUGLASS e colaboradores (17) afirmam ser a panorâmica inferior às técnicas intrabucais nas regiões anteriores.

Os estudos clínicos publicados com relação ao exame radiográfico em periodontia restam infrutíferos, já que não se conhece a verdadeira extensão da patologia, devido às dificuldades de mensuração em alguns sítios e ao grau de variabilidade inter-observadores. (36)

ÅKESSON e ROHLIN (3) observaram que todas as técnicas radiográficas estudadas subestimam a perda óssea alveolar e que as radiografias periapicais pela técnica do paralelismo foram significativamente melhores que a panorâmica e as interproximais, não havendo diferenças significativas entre estas últimas. Neste estudo a variação inter-observadores mostrou-se substancial.

Para RUSHTON e HORNER (53), mesmo não havendo estudos específicos que comparem radiografias intrabucais e panorâmica na visualização do espaço periodontal e da lâmina dura, a experiência clínica aponta para a superioridade das intrabucais. Os autores indicam o uso da panorâmica nos casos de perdas ósseas extensas e na coexistência de outros problemas, salientando a complementação com periapicais quando necessário.

A maioria das lesões periapicais de dentes anteriores não é visualizada na radiografia panorâmica, provavelmente refletindo a perda da qualidade da imagem nesta região devido à superposição da imagem da coluna cervical na mandíbula e do ar presente na cavidade bucal na maxila, na opinião de várois autores. $(1,23,50,58)$

BALIS (7) observou que a panorâmica teve sensibilidade de $79 \%$ e especificidade de $92 \%$ para patologias periapicais. ROHLIN e colaboradores (51) não observaram diferenças significativas entre o desempenho de radiografias panorâmicas e periapicais na evidenciação de lesões apicais, quando analisadas por radiologistas. Em estudo posterior ROHLIN e colaboradores (52) concluíram que a panorâmica foi superior às periapicais quando o grupo de observadores era formado por radiologistas, não tendo sido demonstradas diferenças entre os dois métodos quando os observadores eram clínicos ou endodontistas.

Alguns autores $(39,52)$ não encontraram diferenças significativas entre panorâmica e levantamentos periapicais para o diagnóstico de lesões apicais, enquanto que outros $(43,48)$ concluíram pela superioridade da primeira.

Observa-se na literatura a prática de investigação radiográfica panorâmica de rotina em três grupos bem definidos: pacientes jovens para diagnóstico ortodôntico, adultos dentados e pacientes edêntulos (53).

O uso rotineiro de radiografia panorâmica em pacientes jovens é contestado com base em sua pouca influência no plano de tratamento $(25,42,65)$, contudo, HINTZE e colaboradores (21) salientam que o rendimento diagnóstico varia de acordo com a especialidade do profissional que avalia a radiografia.

A maioria dos estudos sobre o valor da investigação rotineira através de radiografia panorâmica em adultos dentados são análises retrospectivas realizadas em pacientes hospitalares $(5,9,23,29,44,67)$, nas Forças Armadas Americanas $(13,35,38)$, em dentistas voluntários participantes de eventos científicos $(16,32)$ e em estudantes universitários (46).Nestes estudos a proporção de pacientes que apresentou anormalidades variou de $4,8 \%$ (9) a $89,6 \%$ (13); sendo as alterações mais frequientes dentes retidos, cuja a prevalência variou de $1,6 \%$ (9) a $76,6 \%$ (46); doença periodontal, cuja prevalência variou de $1,3 \%(46)$ a $84,4 \%$ (44); e cáries, cuja prevalência variou de $10,8 \%(16)$ a $46,0 \%(46)$.

BARRETT e colaboradores (9) observaram que a investigação panorâmica rotineira de mil pacientes mostrou patologias que implicaram em tratamento imediato em uma pequena proporção deles $(4,8 \%)$.

Muitos estudos têm sido realizados com relação à validade de investigar-se rotineiramente pacientes edêntulos através de radiografias panorâmicas - prática recomendada em 1987 pela Food and Drug Administration. Nestes trabalhos a incidência de anormalidades varia de $0,33 \%$ a $61,00 \%$ dos pacientes. $(5,6,8,18,20,26,30,33$, $34,47,49,54,57)$ Os achados principais estão constituídos por remanescente radiculares, com prevalência variando de $6,0 \%(20)$ a $40,0 \%(8)$; dentes retidos variando de $0,9 \%$ (26) a $10,0 \%$ (49); e presença de corpos estranhos variando entre $0,4 \%$ (6) e $10,0 \%$ dos casos (49). Calcificação de linfonodos e alongamento dos processos estilóides são achados menos frequientes (47).

LYMAN e BOUCHER (34) e GARCIA e colaboradores (20) concluem que a panorâmica de rotina em pacientes edêntulos apresenta pouco valor diagnóstico, e JONES e colaboradores (26) e LLOYD e GAMBERT (33) recomendam a descontinuidade desta prática.

MUIR e GOSS $(40,41)$, estudando tanto indivíduos dentados quanto edêntulos através de radiografias panorâmicas, demonstraram que mais de 90,0\% dos pacientes assintomáticos apresentam algum tipo de alteração radiográfica nas articulações temporomandibulares. Estas alterações consistem em remodelações progressivas, regressivas ou circunferenciais, representadas pela evidenciação radiográfica de osteófitos, erosão ou esclerose das corticais, aplainamento ou concavidade da superfície e ainda cistos subcorticais.

BROOKS e colaboradores (12) afirmam que a panorâmica evidencia alterações do côndilo mandibular e da cavidade articular apenas quando as mesmas são pronunciadas, e que o grau de concordância entre os achados da panorâmica e de tomografias laterais é de 60 a 70,0\%. Já CHEYNET e colaboradores (14) enfatizam que a panorâmica permite um estudo simultâneo e comparativo das articulações temporomandibulares, especialmente quando realizada em oclusão.

A radiografia interproximal é, sem sombra de dúvida, a técnica complementar mais importante da radiografia panorâmica, ainda mais na atualidade, com a moderna odontologia preventiva que diminuiu drasticamente a prevalência de cárie, tornando um ponto de central importância o diagnóstico de cáries proximais incipientes. Esta incidência é utilizada também para visualização da adaptação de restaurações e próteses nas superfícies proximais, evidenciação da existência de tratamento endodônticos nos segmentos posteriores, observação da presença de tártaro nos espaços proximais, existência de maloclusão na região posterior causada por perda de dentes, perda de antagonistas ou contatos prematuros. (45)

Um benefício definido para o profissional que usa como procedimento a requisição de radiografia panorâmica é a aquisição da informação negativa: que cada

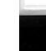


paciente é, de fato, livre de condições patológicas intra-ósseas fora do campo do exame intrabucal. (67)

Analisando a literatura pertinente, pode-se levantar uma série de questionamentos:

- É coerente se iniciar o exame radiográfico de rotina por uma técnica tão específica e de abrangência tão limitada quanto a radiografia interproximal?

- As informações não obtidas na radiografia panorâmica, geralmente lesões incipientes, justificam que não se obtenha informações quanto ao restante das regiões maxilo-mandibulares?

- Existe algum critério que determine que uma cárie proximal (bastante frequiente) seja de maior importância diagnóstica que uma lesão óssea (rara) ou vice-versa?

- Se o custo financeiro de uma radiografia panorâmica e de quatro interproximais se equivalem e a exposição do paciente é menor na radiografia panorâmica, não seria coerente iniciar o exame radiográfico de rotina por uma técnica mais abrangente, partindo do geral para o específico, com radiografias intra-bucais apenas em áreas sugeridas pela radiografia panorâmica, se o diagnóstico não for esclarecido de maneira satisfatória?

Não bastasse todos estes questionamentos, CLIFTON e colaboradores (15) observaram que apesar de apresentar inestimável valor diagnóstico, a radiografia interproximal pode ser de execução bastante desconfortável para o paciente, tecnicamente difícil para o profissional e o número de repetições pode aumentar consideravelmente a dose de exposição.

Recentemente FLINT e colaboradores (19) concluíram que a associação da panorâmica à quatro interproximais equivale em desempenho diagnóstico a um levantamento periapical completo associado a interproximais.

\section{OBJETIVO}

$O$ presente estudo teve por objetivo comparar os diagnósticos obtidos de radiografias interproximais e panorâmica, no sentido de verificar se é possível propor uma mudança na conduta profissional frente à solicitação de exame radiográfico de rotina.

Como estes métodos apresentam áreas de abrangência diversas, num primeiro momento foram comparados os diagnósticos obtidos pelas radiografias interproximais àqueles obtidos pela radiografia panorâmica do mesmo paciente nas áreas correspondentes à interproximal (coroa $\mathrm{e}$ terço cervical das raízes dos dentes molares e pré-molares), para verificar se existem diferenças entre as proporções de diagnósticos coincidentes e divergentes.

Numa segunda etapa foram avaliados os dignósticos fornecidos pela radiografia panorâmica, excluída a área de abrangência das interproximais.

\section{MATERIAIS E MÉTODOS}

Os pacientes selecionados para a presente amostra foram aqueles cujos cirurgiões-dentistas solicitaram pelo menos uma radiografia panorâmica e quatro interproximais (regiões de molares e prémolares, lados direito e esquerdo) com a finalidade de complementação diagnóstica, que procuraram o Centro de Diagnóstico Radiológico para a execução dos exames.

Foram incluídos consecutivamente os primeiros 50 pacientes, de ambos os sexos, que apresentassem idade superior a 15 anos (média de 32 anos), considerados adultos conforme BOHAY e colaboradores (10), que concordaram em participar da pesquisa, de acordo com o Termo de Consentimento Informado.

Para realização da radiografia panorâmica foi utilizado o aparelho Odontorama PC (Trophy Radiologie, MarneLa-Vallée, França) com fatores de exposição variáveis ( 55 a $100 \mathrm{kV}$ e 3 a $10 \mathrm{~mA})$, filmes $15 \times 30 \mathrm{~cm}$ T-Mat Kodak em chassis providos de écrans Lanex Medium (Eastman Kodak, Rochester, EUA).

O exame foi realizado com o paciente sentado, com a cabeça fixa em cefalostato de apoios temporais, de maneira que o plano médio sagital ficasse perpendicular ao solo e o plano horizontal de Frankfurt paralelo ao solo, ocluindo em topo com o auxílio de um mordedor.

As radiografias interproximais foram executadas em um aparelho Spectro II (DabiAtlante, Ribeirão Preto, SP) com $50 \mathrm{kVp}$ e $8 \mathrm{~mA}$, variando-se o tempo de exposição, e filmes kodak Ektaspeed Plus $\mathrm{n}^{\circ} 2$ (Eastman Kodak, Rochester, EUA), com o auxúlio de posicionadores interproximais (Jon, São Paulo) ou de aletas de mordida (Crosstex, Nova Iorque, EUA).

Para o processamento dos filmes foi utilizada processadora automática modelo $\mathrm{A} /$ T 2020 (All-Pro Imaging Corp., Hicksville, EUA) e químicos X-Omat (Eastman Kodak, Rochester, EUA).

Os cirurgiões-dentistas que solicitaram as radiografias dos pacientes selecionados foram contactados e thes foi solicitada a disponibilização dos exames para a coleta de dados.

O exame das radiografias foi realizado sempre pelo mesmo observador, especialista em radiologia odontológica, em momentos diferentes (primeiro todas as panorâmicas e após um mês todas as interproximais), sob condições ideais de interpretação: em ambiente escurecido, utilizando lupa e negatoscópio provido de máscara.

Os diagnósticos radiográficos seguiram critérios pré-estabelecidos e foram anotados por dente, paciente e por método radiográfico, em fichas específicas para tal finalidade, arquivadas em meio eletrônico.

Após examinada, cada radiografia foi digitalizada através de scanner com adaptador de transparência (Hewlett-Packard, Palo Alto, EUA), arquivada em meio eletrônico e então devolvida ao cirurgião-dentista que a solicitou.

Para a análise estatística os diagnósticos foram substituídos por códigos numéricos. Inicialmente foram comparados os diagnósticos obtidos pelas radiografias interproximais em todos os molares e prémolares com aqueles das panorâmicas nos dentes respectivos, de todos os pacientes. $\mathrm{Ou}$ seja, avaliou-se os diagnósticos obtidos pelos dois métodos exclusivamente nas regiões abrangidas pela técnica interproximal (coroa e terço cervical das raízes de molares e prémolares). Para avaliar se existem diferenças entre ambos os métodos utilizou-se a Prova Binomial.

A seguir, os diagnósticos obtidos pela panorâmica nas regiões não registradas na técnica interproximal foram listados.

\section{RESULTADOS}

Dos 50 pacientes examinados 29 pertencem ao sexo feminino $(58,0 \%)$ e 21 ao sexo masculino $(42,0 \%)$, com idade variando entre 15 e 66 anos. A média de idade para ambos os sexos foi de 32,22 anos, sendo 31,03 anos para o sexo feminino e 33,86 anos para o sexo masculino.

Os diagnósticos obtidos em ambas as técnicas estão listados e quantificados no quadro 01. Considera-se como área excedente as regiões compreendidas na radiografia panorâmica que excedem os limites das interproximais - dentes incisivos e caninos superiores e inferiores, terços médio e apical das rázes dos molares e pré-molares, tecido ósseo, regiões temporomandibulares e seios maxilares.

A tabela 1 compara os diagnósticos obtidos através dos dois métodos radiográficos na área de abrangência das radiografias interproximais.

Através da Prova Binomial, que objetiva verificar a existência de diferenças entre duas proporções obtidas de uma amostra dividida em duas classes (isto é, dicotomizadas: diagnósticos coincidentes ou não coincidentes), verifica-se que há 
diferenças estatísticas entre a proporção de dentes com diagnósticos coincidentes e divergentes. Observou-se que a grande maioria dos dentes $(97,8 \%)$ apresentaram diagnósticos coincidentes, o que sugere não haver diferença entre os dois tipos de exames, quanto ao rendimento diagnóstico.

A tabela 2 mostra as divergências diagnósticas observadas entre ambos os métodos na área de abrangência das radiografias interproximais.

A tabela 3 mostra os diagnósticos observados na área de abrangência das radiografias interproximais que foram observados somente na panorâmica. Dos 4 elementos dentários considerados ausentes nas interproximais, observou-se na panorâmica que 2 encontravam-se não irrompidos, 1 retido e 1 apresentava remanescentes radiculares. Nos 2 casos restantes, a diferença se constituiu na observação de cáries não diagnosticadas pelas interproximais.

Os diagnósticos observados somente nas radiografias interproximais são mostrados na tabela 4. Aqui observa-se que a radiografia panorâmica não mostrou a presença de 6 cáries, a ocorrência de 4 reincidências de cárie sob restaurações (3 em amálgama e 1 estética), 3 má adaptações de restaurações em amálgama, 1 perfuração no terço cervical de raiz e a presença de tártaro em 2 dentes.

Os diagnósticos obtidos de alterações na área excedente da radiografia panorâmica estão relacionados no quadro 2 .

A radiografia panorâmica propiciou 792 diagnósticos que não poderiam ser obtidos pelas interproximais. Destes, 606 $(76,51 \%)$ não implicam na necessidade de tratamento, tendo caráter exclusivamente informativo.

Contudo, os 186 (23,49\%) diagnósticos restantes (média de 3,72 por paciente) implicam na:necessidade de tratamento ou controle. Dentre eles preponderaram os problemas endodônticos, seguidos de alterações na coroa dos dentes anteriores.

Alterações na forma e ou superfície dos côndilos mandibulares, bem como lesões ósseas, foram observações pouco expressivas. Não foram diagnosticadas patologias sinusais.

\section{DISCUSSÃO}

Foram incluídos na presente amostra pacientes com idade igual ou superior a 15 anos, por se concordar com BOHAY e colaboradores (10) quanto ao fato de que a partir dos 15 anos o paciente não é mais considerado pediátrico.

Todos os pacientes desta amostra foram aqueles cujos cirurgiões-dentistas consideraram adequado solicitar uma radiografia panorâmica e quatro interproximais para a complementação do exame clínico, uma combinação de exames frequientemente observada por KAUGARS e colaboradores (28).

Foram respeitados todos os procedimentos quanto à padronização das incidências e do processamento radiográfico, por se estar de acordo com SCHIFF e colaboradores (1986), BREZDEN e BROOKS (1987), ÅKESSON e colaboradores (1991) e SMITH e colaboradores (1993), quando citam que estes fatores estão intimamente ligados à qualidade das radiografias.

Todas as radiografias foram examinadas pelo mesmo observador, visto estar comprovado que tanto radiografias panorâmicas (51) quanto intrabucais (52) apresentam diagnósticos mais precisos se analisadas por radiologistas, considerandose que a variação inter-observadores é expressiva (3) e que o rendimento diagnóstico varia de acordo com a especialidade e a experiência do profissional que avalia a radiografia (21).

No sentido de evitar que uma imagem observada na radiografia interproximal fosse lembrada pelo intérprete no momento da avaliação da panorâmica, primeiro foram examinadas as radiografias panorâmicas e, após transcorrido um mês, todas as interproximais.

Os dentes caninos foram excluídos da área considerada de abrangência das radiografias interproximais por não mostrarem imagem satisfatória de toda e coroa e do terço cervical da raiz em todos os pacientes.

Com subsídios nos achados de MOLANDER e colaboradores (36) e de ÅKESSON e ROHLIN (3), nos quais tanto radiografias interproximais quanto panorâmicas subestimaram a perda óssea alveolar e não diferiram significativamente entre si, na presente amostra não foram considerados os diagnósticos radiográficos relacionados à crista alveolar.

$\mathrm{O}$ presente estudo difere de muitos trabalhos encontrados na literatura $(5,9,13$, $16,23,29,32,35,38,39,44,46,67)$, já que não tem por objetivo avaliar se é ou não justificável a solicitação rotineira de radiografia panorâmica para a população em geral.

Também não se busca avaliar qual das técnicas radiográficas é mais adequada ao diagnóstico de patologias dentoalveolares específicas, como fizeram alguns autores para cáries $(1,7,17,24,31,37,43,59,60)$, para doença periodontal $(2,3,17,22,27$, $44,36,37,53)$ e para alterações periapicais

\section{$(1,7,23,39,43,48,50,52,58)$}

Ao contrário, avalia-se ambas as técnicas em termos globais de diagnóstico odontológico, ou seja, que informações são perdidas ou ganhas quando se opta por uma das duas técnicas, estudo que não encontra paralelo na literatura.

A proporção de diagnósticos coincidentes em ambas as técnicas para a área de abrangência das radiografias interproximais foi fortemente significativa, pois em apenas $2,2 \%$ dos dentes examinados houveram diferenças: 1,6\% dos diagnósticos foram considerados superiores na interproximal e $0,6 \%$ na panorâmica.

As radiografias panorâmicas não permitiram uma média de 0,32 diagnósticos por paciente. As informações perdidas nesta técnica constituíram-se basicamente na presença de cáries e reincidências de cáries. Por outro lado, as radiografias interproximais também apresentaram menor rendimento diagnóstico: quatro dentes considerados ausentes estavam retidos, não-erupcionados ou apresentavam raízes residuais e duas cáries não foram diagnosticadas. A análise desses seis casos sugere que problemas no posicionamento do filme e na angulagem horizontal prejudicaram o diagnóstico. Estas dificuldades inerentes à técnica já foram demonstradas por CLIFTON e colaboradores (15) e por VAN DER STELT e colaboradores (63).

Além da área de abrangência das radiografias interproximais a panorâmica propiciou 186 diagnósticos (média de 3,72 por paciente) que implicam na necessidade de tratamento ou controle. Dentre estes diagnósticos, preponderaram os problemas endodônticos, que certamente seriam adequadamente demostrados através de radiografias periapicais $(39,43,48)$, porém às custas de um considerável aumento na exposição à radiação.

As alterações temporomandibulares, apesar de constituírem um pequeno percentual no universo de diagnósticos adicionais permitidos pela panorâmica, atingiram $20,0 \%$ dos pacientes da presente amostra, percentual inferior ao encontrado por MUIR e GOSS $(40,41)$ e por BROOKS e colaboradores (12).

Desta forma, a significativa coincidência de diagnósticos obtidos à partir de ambas as técnicas radiográficas na presente amostra; a relativa facilidade e comodidade de execução da panorâmica em relação às interproximais (15); a equivalência no custo de uma panorâmica e de quatro interproximais; e, principalmente, a relativamente baixa dose de exposição à radiação durante a radiografia panorâmica 
quando são utilizados equipamentos mais recentes (66), permitem que se considere indicada a substituição das radiografias interproximais pela panorâmica naqueles pacientes em início de tratamento, nos quais o exame clínico necessite de complementação radiográfica.

\section{CONCLUSÕES}

Os resultados encontrados neste estudo permitem concluir que:

a) não existe diferença estatisticamente significativa entre o rendimento diagnóstico de radiografias panorâmica e interproximais, na área de abrangência desta última;

b) além da área de abrangência das radiografias interproximais, a panorâmica mostrou, para a média dos pacientes, 3,72 diagnósticos que requerem tratamento ou controle. Isto nos permite considerar a radiografia panorâmica como uma alternativa às radiografias interproximais na complementação do exame clínico de pacientes em início de tratamento.

\section{SUMMARY}

Our pourpose was to compare the radiographic diagnostics observed in bitewing and panoramic techniques in a sample of 50 adult patients' $x$-rays, examined by the same observer. On the panoramic it was analyzed the area corresponding bitewings, looking for coincident diagnostics. Furthermore it was evaluated the additional information supplied by panoramic technique. The proportion of coincident diagnostics in panoramic and bitewings was significantly larger suggesting no differences between bitewings and panoramic as a diagnostic resource. When analyzing the area not inclued by bitewings on the panoramic it was found 3,72 diagnostics per patient that would request treatment or follow-up.

\section{KEYWORDS}

Dental radiology - Bitewing $x$-ray Panoramic x-ray

\section{REFERÊNCIAS BIBLIOGRÁFICAS}

1. AHLQWIST, M; HALLING, A.; HOLLENDER, L. Rotational panoramic radiography in epidemiological studies of dental health: comparison between panoramic radiographs and intraoral full mouth surveys. Swed. Dent. J., Jonkoping, v. 10, n. 1-2, p. 73-84, 1986.

2. AINAMO J.; TAMMISALO, E. H. Comparison of radiographic and clinical signs of early periodontal disease. Scand. $\mathbf{J}$. Dent.. Res., Copenhagen, v. 81, n. 7, p. 548-552, 1973.
3. ÅKESSON, L.; ROHLIN, M. Comparison of panoramic and intraoral radiography and pocket probing for the measurement of the marginal bone level. $\mathbf{J}$. Clin. Periodontol., Copenhagen, v. 19, n. 5, p. 326-332, May 1992.

4. ÅKESSON, L. et al. An evaluation of image quality for the assessment of the marginal bone level in panoramic radiography. Swed. Dent. J., Malmo, Suppl. 78, p. 101-129, 1991.

5. ALATTAR, M. M.; BAUGHMAN, R. A.; COLLETT, W. K. A survey of panoramic radiographs for evaluation of normal and pathologic findings. Oral Surg. Oral Med. Oral Pathol., Saint Louis, v. 50 , n. 5, p. 472-478, Nov. 1980.

\section{AXELSSON,}

Orthopantomographic examination of the edentulous mouth. J. Prosthet. Dent., Saint Louis, v. 59, n. 5, p. 592-598, May 1988.

7. BALIS, S. Error and accuracy rates of panoramic radiography as a screening method for mass surveying of children. $\mathbf{J}$. Public Health Dent., Richmond, v. 41, n. 4, p. 220-234, Fall 1981.

8. BARCLAY, J. K.; DONALDSON, K. I. Panoramic radiography of the edentulous jaws - a survey of 100 patients. N. Z. Dent. J., Auckland, v. 66, n. 303, p. 53-60, Jan. 1970.

9. BARRETT, A. P.; WATERS, B. E.; GRIFFITHS, C. J. A critical evaluation of panoramic radiography as a sreening procedure in dental practice. Oral Surg. Oral Med. Oral Pathol., Saint Louis, v. 57 , n. 6, p. 673-677, June 1984.

10. BOHAY, R. N.; STEPHENS, R. G.; KOGON, S. L. Survey of radiographic practices of general dentists for the dentate adult patient. Oral Surg. Oral Med. Oral Pathol. Oral Radiol. Endod., Saint Louis, v. 79, n. 4, p. 526-531, Apr. 1995.

11. BREZDEN, N. A.; BROOKS, S. L. Evaluation of panoramic dental radiographs taken in private practice. Oral Surg. Oral Med. Oral Pathol., Saint Louis, v. 63, n. 5, p. 617-621, May 1987.

12. BROOKS, S. et al. Imaging of the temporomandibular joint: a position paper of the American Academy of
Oral and Maxillofacial Radiology. Oral Surg. Oral Med. Oral Pathol. Oral Radiol. Endod., Saint Louis, v. 83, n. 5, p. 609. 618, May 1997.

13. BURGESS, J. O. A panoramic radiographic analysis of Air Force basic trainees. Oral Surg. Oral Med. Oral Pathol., Saint Louis, v. 60, n. 1, p. 113 117, July 1985.

14. CHEYNET, F. et al. Apport des radiographies standard dans le bilan des dysfonctionnements de l'appareil manducateur (D.A.M.). Rev. Stomatol. Chir. Maxillofac., Paris, v. 99, n. 2, p. 88102, Juin 1998.

15. CLIFTON, T. L.; TYNDALL, D. A.; LUDLOW, J. B. Extraoral radiographic imaging of primary caries. Dentomaxillofac. Radiol., Houndsmills, v. 27, n. 4, p. 193-198, july 1998.

16. CUTTINO, C. L. et al. Panoramic radiographic survey of dentists: interpretation of findings. J. Am. Dent. Assoc., Chicago, v. 79, n. 5, p. 1179-1182, Nov. 1969.

17. DOUGLASS, C. W. et al. Clinical efficacy of dental radiography in the detection of dental caries and periodontal diseases. Oral Surg. Oral Med. Oral Pathol., Saint Louis, v. 62, n. 3, p. 330 339, Sept. 1986.

18. EDGERTON, M.; CLARK, P. Location of abnormalities in panoramic radiographs of edentulous patients. Oral Surg. Oral Med. Oral Pathol., Saint Louis, v. 71, n. 1, p. 106109, Jan. 1991.

19. FLINT, D. J. et al. A diagnostic comparison of panoramic and intraoral radiographs. Oral Surg. Oral Med. Oral Pathol. Oral Radiol. Endod., Saint Louis, v. 85, n. 6, p.731-735, June 1998.

20. GARCIA, R. I.; VALACHOVIC, R. W.; CHAUNCEY, H. H. Longitudinal study of the diagnostic yield of panoramic radiographs in aging edentulous men. Oral Surg. Oral Med. Oral Pathol. Saint Louis, v. 63, n. 4, p. 494-497, Apr. 1987.

21.

HINTZE, H.; WENZEL,

A.; WILLIAMS, S. Diagnostic value of clinical examination for the identification of children in need of orthodontic treatment compared 
with clinical examinations and screening pantomography. Eur. J. Orthod., Oxford, v. 12 , n. 4, p. 385-388, Nov. 1990.

22. HIRSCHMAN, P. N. Radiographic interpretation of chronic periodontitis. Int. Dent. J., Guildford, v. 37, n. 1, p. 3-9, Mar. 1987.

23. HORTON, P. S. et al. Analysis of interpretations of full-mouth and panoramic surveys. Oral Surg. Oral Med. Oral Pathol., Saint Louis, v. 44, n. 3, p. 468-475, Sept. 1977.

24.

HURLBURT, C. E.; WUEHRMANN, A. H. Comparison of interproximal carious lesion detection in panoramic and standard intraoral radiography. J. Am. Dent. Assoc., Chicago, v. 93, n. 6, p. 1154-1158, Dec. 1976.

25. IGNELZI, M. A.; FIELDS, H. W.; VANN, W. F. Screening panoramic radiographs in children: prevalence data and implications. Pediatr. Dent., Chicago, v. 11, n. 4, p. 279-285, Dec. 1989

26. JONES, J. D.; SEALS, R. R.; SCHELB, E. Panoramic radiographic examination of edentulous patients. J. Prosthet. Dent., Saint Louis, v. 53, n. 4, p. 535-539, Apr. 1985.

27. KAIMENYI, J. T.; ASHLEY, F. P. Assessment of bone loss in periodontitis from panoramic radiographs. J. Clim. Periodontol., Copenhagen, v. 15, n. 3, p. 170- 174, Mar. 1988.

28. KAUGARS, G. E.; BROGA, D. W.; COLLETT, W. K. Dental radiologic survey of Virginia and Florida. Oral Surg. Oral Med. Oral Pathol, Saint Louis, v. 60, n. 2, p. 225-229, Aug 1985.

29. KEITH, D. A. The detection of abnormalities in the jaws: a survey. Br. Dent. J., London, v.' 134, n. 4, p. 129-135, Feb. 1973.

30. KEUR, J. J.; CAMPBELL, J. P. S.; MC CARTHY, J. F. Radiological findings in 1135 edentulous patients. J. Oral Rehabil., Oxford, v. 14, n. 2, p. 183-191, Mar. 1987.

31. KIDD, E. A. M.; PITTS, N. B. A reappraisal of the value of the bitewing radiograph in the diagnosis of posterior approximal caries. Br. Dent. J.,
London, v. 169, n. 7, p. 195-200, Oct. 1990.

32.

LANGLAND, O. E. et al.

Panoramic radiographic survey of dentists participating in ADA health screening programs: 1976, 1977, and 1978. J. Am. Dent. Assoc., Chicago, v. 101, n. 2, p. 279282, Aug. 1980.

\section{LLOYD, P. M.;} GAMBERT, S. R. Periodic oral examinations and panoramic radiographs in edentulous elderly men. Oral Surg. Oral Med. Oral Pathol., Saint Louis, v. 57, n. 6, p. 678680, June 1984.

\section{LYMAN, S.; BOUCHER,} L. J. Radiographic examination of edentulous mouths. J. Prosthet. Dent., Saint Louis, v. 64, n. 2, p.180-182, Aug. 1990.

35.

MEISTER, F.; SIMPSON,

J.; DAVIES, E. E. Oral health of airmen: analysis of panoramic radiographic and Polaroid photographic survey. J. Am. Dent. Assoc., Chicago, v. 94, n. 2, p. 335-339, Feb. 1977.

36.

MOLANDER, B. et al. Agreement between panoramic and intra-oral radiography in the assessment of marginal bone heigth. Dentomaxillofac. Radiol., Houndsmills, v. 20, n. 3, p. 155-160, Aug. 1991.

37.

MOLANDER, B. et al. Comparison of panoramic and intraoral radiography for the diagnosis of caries and periapical pathology. Dentomaxillofac. Radiol., Houndsmills, v. 22, n. 1, p. 28-32, Feb. 1993.

38. MORRIS, C. R. et al. Abnormalities noted on panoramic radiographs. Oral Surg. Oral Med. Oral Pathol., Saint Louis, v. 28, n. 5, p. 772 782, Nov. 1969.

39. MUHAMMED, A. H; MANSON-HING, L. R. A comparison of panoramic and intraoral radiographic surveys in evaluating a dental clinic population. Oral Surg. Oral Med. Oral Pathol., Saint Louis, v. 54, n. 1, p. 108-117, July 1982.

40. MUIR, C. B.; GOSS, A. The radiologic morphology of asymptomatic temporomandibular joints. Oral Surg. Oral Med. Oral Pathol., Saint Louis, v. 70, n. 3, p. 349-354, Sept. 1990a.

41. MUIR, C. B.; GOSS, A.
The radiologic morphology of painful temporomandibular joints. Oral Surg. Oral Med. Oral Pathol., Saint Louis, v. 70, n. 3, p. 355-359, Sept. 1990b.

42. MYERS, D. R. et al. Radiographic recommendations for the transitional dentition: comparison of general dentists and pediatric dentists. Pediatr. Dent., Chicago, v. 12, n. 4, p. 217-221, July/Aug. 1990.

43.

OHBA, T.; KATAYAMA, H. Comparison of orthopantomography with conventional periapical dental radiography. Oral Surg. Oral Med. Oral Pathol., Saint Louis, v. 34, n. 3, p. 524-530, Sept. 1972.

44.

OSBORNE, G. E.; HEMMINGS, K. W. A survey of disease changes observed on dental panoramic tomographs taken of patients attending a periodontology clinic. Br. Dent. J., London, v. 173, n. 5, p. 166-168, Sept. 1992.

45.

PASLER, F. A Radiologia odontológica. 3.ed. São Paulo : Medsi, 1999.

46. PELTOLA, J. S. A panoramatomographic study of the teeth and jaws of Finnish university students. Community Dent. Oral Epidemiol., Copenhagen, v. 21, n. 1, p. 36-39, Feb. 1993.

47.

PERRELET, L. A.; BERNHARD, M.; SPIRGI, M. Panoramic radiography in the examination of edentulous patients. J. Prosthet. Dent., Saint Louis, v. 37, n. 5, p. 494-498, May 1977.

48.

PHILLIPS, J. D.; SHAWKAT, A. H. A study of the radiographic appearance of osseous defects on panoramic and conventional films. Oral Surg. Oral Med. Oral Pathol., Saint Louis, v. 36, n. 5, p. 745-749, Nov. 1973.

49.

RITCHIE, G. M.; FLETCHER, A. M. A radiographic investigation of edentulous jaws. Oral Surg. Oral Med. Oral Pathol., Saint Louis, v. 47, n. 6, p. 563-567, June 1979.

50. ROHLIN, M.; ÅKERBLON, A. Individualized periapical radiography determined by clinical and panoramic examination. Dentomaxillofac. Radiol., Houndsmills, v. 21, n. 3, p.135141, Aug. 1992. 
51.

ROHLIN, M. et al. Comparison between panoramic and periapical radiography in the diagnosis of periapical bone lesions. Dentomaxillofac. Radiol., Houndsmills, v. 18, n. 4, p. 151155, Nov. 1989.

52.

ROHLIN, M. et al. Observer performance in the assessment of periapical pathology: a comparison of panoramic with periapical radiography. Dentomaxillofac. Radiol., Houndsmills, v. 20, n. 3, p.127-131, Aug. 1991.

53. RUSHTON, V. E.; HORNER, K. The use of panoramic radiology in dental practice. J. Dent., Kidlington, v. 24, n. 3, p. 185-201, May 1996.

54. SCANDRETT, F. R. et al. Radiographic examination of the edentulous patients. Part I: Review of the literature and preliminary report comparing trhee methods. Oral Surg. Oral Med. Oral Pathol., Saint Louis, v. 35, n. 2, p. 266-274, Feb. 1973.

55. SCHIFF, T. et al. Common positioning and technical errors in panoramic radiography. J. Am. Dent. Assoc., Chicago, v. 113, n. 3, p. 422-426, Sept. 1986.

56. SMITH, N. J. D. et al. Assessment of the quality of panoramic radiographs taken in general dental practice. J. Dent. Res., Alexandria, v. 72, n. 4, p. 712, Apr. 1993. Abstract 204.

57. SPYROPOULOS, N. D.; PATSAKAS, A. J.; ANGELOPOULOS, A. $P$. Findings from radiographs of the jaws of edentulous patients. Oral Surg. Oral Med. Oral Pathol., Saint Louis, v. 52, n. 4, p. 455-459, Oct. 1981.

58.

STEPHENS, R. G. et al.

A comparison of Panorex and intraoral surveys for routine dental radiography. Dent. J., Ottawa, v. 43, n. 6, p. 281-286, June 1977.

59.

STEWART, J. L.;

BIESER, L. F. Panoramic roentgenograms compared with conventional intraoral roentgenograms. Oral Surg. Oral Med. Oral Pathol., Saint Louis, v. 26, n. 1, p. 39-42, July 1968.

60. SWAN, E. S. C.; LEWIS, D. W. Ontario dentists: 1. Radiological practices and opinions. J. Can. Dent. Assoc., Toronto, v. 59, n. 1, p. 62-67, Jan.
1993.

61.

VALACHOVIC, R. W.; LURIE, A. G. Risk-benefit considerations in pedodontic radiology. Pediatr. Dent., Chicago, v. 2, n. 2, p. 128-146, June 1980.

62. VALACHOVIC, R. W. et al. The use of panoramic radiography in the evaluation of asymptomatic adult dental patients. Oral Surg. Oral Med. Oral Pathol., Saint Louis, v. 61, n. 3, p. 289296, Mar. 1986.

63.

VAN DER STELT, P. F. et al. In vitro study into the influence of $x$-ray beam angulation on the detection of artificial caries defects on bite-wing radiographs. Caries Res., Basel, v. 23, n. 5, p. 334-341, 1989.

64.

WALL, B. F.; KENDAL, G. M. Collective doses and risks from dental radiology in Great Britain. Br. J. Radiol., London, v. 56, n. 668, p. 511-516, Aug. 1983.

65. WENZEL, A. Radiographic screening for identification of children in need of orthodontic treatment? Dentomaxillofac. Radiol., Houndsmills, v. 20, n. 3, p. 115-116, Aug. 1991.

66. WHITE, S. C. Assessment of radiation risk from dental radiography. Dentomaxillofac. Radiol., Houndsmills, v. 21, n. 3, p. 118-126, Aug. 1992.

67.

WHITE, S. C.; WEISSMAN, D. D. Relative discernment of lesions by intraoral and panoramic radiography. J. Am. Dent. Assoc., Chicago, v. 95, n.. 6, p. 1117-1121, Dec. 1977.

\footnotetext{
Endereço para Correspondência:

Vania Fontanella

Rua Ramiro Barcelos, 2492

Porto Alegre - RS

0-XX-51.316.5199

e-mail: vaniafontanella@zaz.com.br
} 
TABELA 1 - Comparação entre os diagnósticos obtidos pelas técnicas panorâmica e interproximal n área de abrangência desta última, Porto Alegre, 1999.

\begin{tabular}{c|c|c|c}
\hline COMPARAÇÃ O & $N^{\circ}$ DENTES & \% DENTES & $p$ \\
\hline Diagnósticos coincidentes & 978 & 97,8 & $0,0000^{*}$ \\
Diagnósticos divergentes & 22 & 2,2 & \\
\hline & & & \\
TOTAL & 1000 & 100 & - \\
\hline
\end{tabular}

Hipóteses de Teste: $\quad$ pl: proporção de diagnósticos coincidentes

p2: proporção de diagnósticos divergentes

H0: $\mathrm{p} 1=\mathrm{p} 2$

$\mathrm{H} 1: \mathrm{p} 1>\mathrm{p} 2$

TABELA 2 - Divergências disgnósticas entre os dois métodos na área de abrangência das radiografi: interproximais, Porto Alegre, 1999.

\begin{tabular}{c|c|c}
\hline DIAGNÓSTICO & $\boldsymbol{N}^{\circ}$ DENTES & \% DENTES \\
\hline Observado somente na Panorâmica & 6 & 27,27 \\
Observado somente na Interproximal & 16 & 72,73 \\
\hline TOTAL & 22 & $\mathbf{1 0 0 , 0 0}$ \\
\hline
\end{tabular}

TABELA 3 - Diagnósticos na área de abrangência das radiografias interproximais que foram obsern. dos somente na panorâmica, Porto Alegre, 1999.

\begin{tabular}{c|ccccc}
\hline \multirow{2}{*}{ INTERPROXIMAL } & & & PANORÂMICA \\
\cline { 2 - 5 } & 3 & -4 & 5 & $10+9$ & $10+9+7$ \\
\hline 1 & 1 & - & - & - & - \\
10 & - & - & - & - & - \\
$10+7$ & - & & & \\
\hline
\end{tabular}


TABELA 4 - Diagnósticos que foram observados nas radiografias interproximais, Porto Alegre, 1999.

\begin{tabular}{|c|c|c|c|c|c|}
\hline \multirow[t]{2}{*}{ INTERPROXIMAL } & \multicolumn{5}{|c|}{ PANORÂMICA } \\
\hline & 2 & 10 & 11 & $11+4$ & $12+3$ \\
\hline 9 & 1 & - & - & - & - \\
\hline $10+7$ & - & 1 & - & - & - \\
\hline $10+9$ & - & 4 & - & $\therefore$ & - \\
\hline $10+13$ & - & 3 & - & - & - \\
\hline $10-14$ & - & 3 & - & - & - \\
\hline $11-14$ & - & - & 1 & - & - \\
\hline $11-17$ & - & - & 1 & - & - \\
\hline $11-14+9$ & - & - & - & 1 & - \\
\hline $12-13+7$ & - & - & - & - & 1 \\
\hline
\end{tabular}

Legenda: 2. Hígido, 7. Tártaro, 9. Cárie, 10. Restauraçao Metálica, 11. Restauração Estética, 12. Coroa protética, 13. Má adaptação de restauração ou coroa protética, 14. Reincidência de cárie, 17. Perfuração 
TABELA 1 - Diagnóstico obtidos pelas duas técnicas, Porto Alegre, 1999.

\begin{tabular}{|c|c|c|c|}
\hline & \multicolumn{2}{|c|}{$\begin{array}{l}\text { Área de abrangências das } \\
\text { radiografias interproximais }\end{array}$} & \multirow{2}{*}{$\begin{array}{c}\text { Área excedente } \\
\text { Número de } \\
\text { ocorrências nas } \\
\text { radiografias } \\
\text { panorâmicas }\end{array}$} \\
\hline Diagnósticos & $\begin{array}{l}\text { Número de } \\
\text { ocorrências nas } \\
\text { radiografias } \\
\text { interproximais }\end{array}$ & $\begin{array}{l}\text { Número de } \\
\text { ocorrências nas } \\
\text { radiografias } \\
\text { panorâmicas }\end{array}$ & \\
\hline Ausente & $179 *$ & 175 & 4 \\
\hline Hígido & 227 & 227 & 417 \\
\hline Retido & 15 & $16^{*}$ & 0 \\
\hline Não irrompido & 13 & $15^{*}$ & 0 \\
\hline Remanescer radicular & 3 & $4^{*}$ & 2 \\
\hline Lesão apical & 5 & 5 & 59 \\
\hline Tártaro & 36 & 34 & 13 \\
\hline Impactado & 1 & 1 & 0 \\
\hline Cárie & $39 * *$ & $35 * *$ & 3 \\
\hline Restauração metálica & 413 & 413 & 14 \\
\hline Restauração estética & 45 & 45 & 110 \\
\hline Coroa protética & 87 & 87 & 46 \\
\hline Má adaptação de restauração ou coroa protética & 49 & 46 & 4 \\
\hline Reincidência de cárie sob restauração ou coroa protética & 70 & 66 & 15 \\
\hline Osteoesclerose apical & 0 & 0 & 11 \\
\hline Destruição da coroa & 8 & 8 & 1 \\
\hline Perfuração & 2 & 1 & 0 \\
\hline Aumento do espaço periodontal apical & 0 & 0 & 26 \\
\hline Rizotomia & 1 & 1 & 0 \\
\hline Falha na onturação endodôntica & 2 & 2 & 43 \\
\hline Separação de raízes & 1 & 1 & 0 \\
\hline Área radiolúcida não associada a dente & 0 & 0 & 2 \\
\hline Instrumento endodôntico fraturado & 0 & 0 & 1 \\
\hline Excesso de material obturador endodôntico & 0 & 0 & 1 \\
\hline Bifurcação de condutos & 0 & 0 & 1 \\
\hline Dente supranumerário retido & 0 & 0 & 2 \\
\hline Obturação retrógrada & 0 & 0 & 1 \\
\hline Reabsorção dentinária externa & 0 & 0 & 1 \\
\hline Pino intra-canal sem endodontia & 0 & 0 & 5 \\
\hline Alterações dos seios maxilares & 0 & 0 & 0 \\
\hline Alteração de côndilo unilateral & 0 & 0 & 5 \\
\hline Alteração de côndilo bilateral & 0 & 0 & 5 \\
\hline
\end{tabular}

* 4 dentes considerados ausentes nas radiografias interproximais foram diagnosticados como não irrompodos (2), retido (1) e remanescente radicular . panorâmica.

** Foram diagnosticadas ao total 41 cáries, das quais 6 só foram observadas nas radiografias interproximais e 2 somente na panorâmica. 
TABELA 2 - Diagnósticos obtidos na radiografia panorâmica, excluídos aqueles possíveis de serem observados na área de abrangência das interproximais, Porto Alegre, 1999.

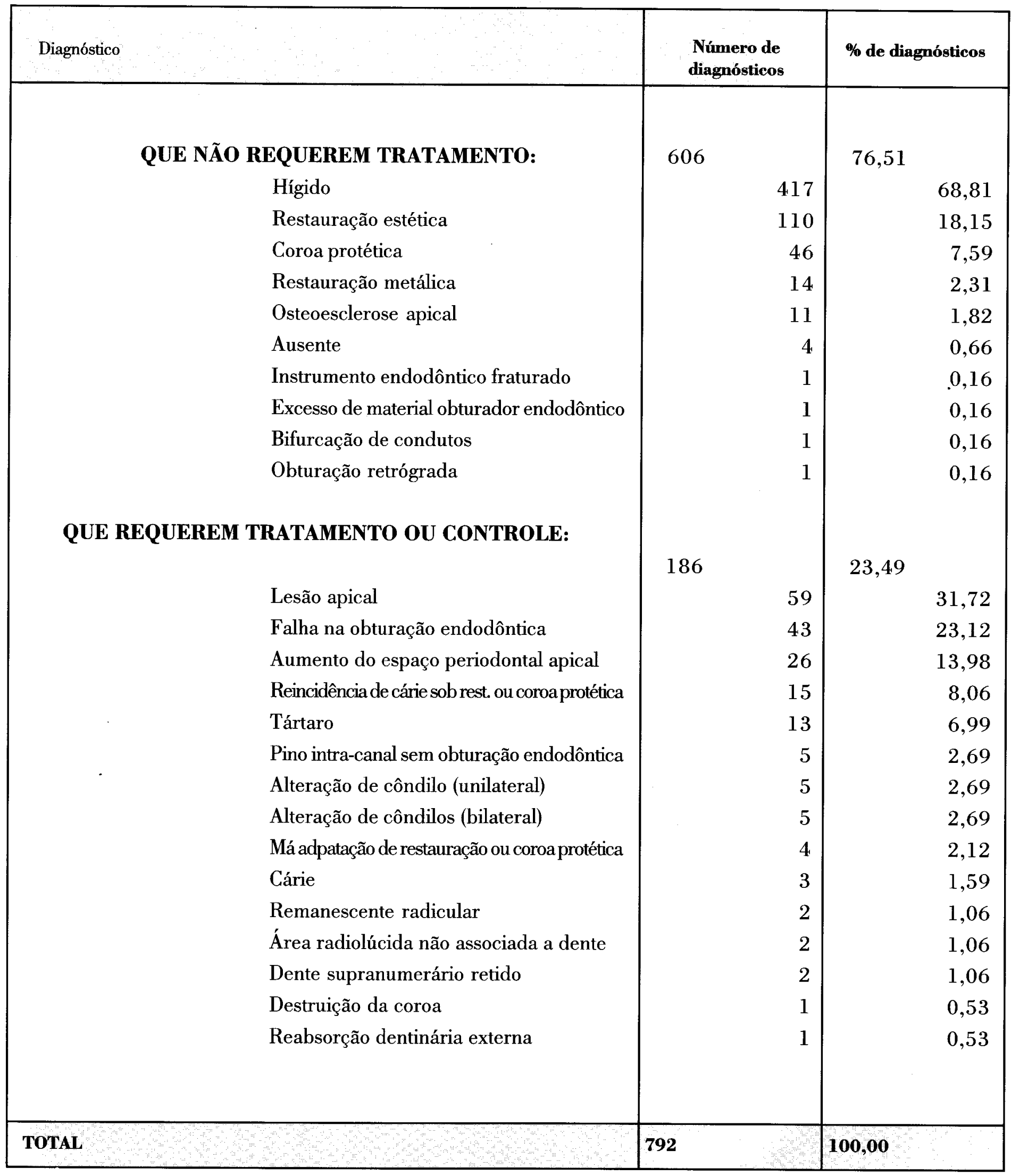

